\title{
Autonomous magnetic devices for micro/nano particle handling
}

\author{
Luiz F. ZANINI ${ }^{1,2}$, Frédéric DUMAS-BOUCHIAT ${ }^{1}$
}

\author{
1- Institut Néel, CNRS UPR 2940 \& Univ. J. Fourier, BP 166, 38042 Grenoble Cedex 9, France \\ 2- G2Elab, CNRS UMR5269, MINATEC-CIME, 3 parvi L. Néel, 38016 Grenoble Cedex 1, France
}

\begin{abstract}
This article describes a lab practical based on the fabrication of thick hard magnetic films, their patterning at the micro-scale, their integration into microfluidic systems and their use in particle capture and sorting. It combines both standard and recently developed microfabrication techniques in order to create autonomous, downscaled magnetic micro-systems which can produce high magnetic forces on micro- and nano-sized objects. The lab practical was designed for Master students, but can be used as an introductory course for higher-level students starting in the field.
\end{abstract}

\section{Introduction}

This lab practical is divided in three parts. The first part consists of developing thick, hard magnetic films by triode sputtering, a Physical Vapor Deposition (PVD) technique. The deposition of the film is followed by its magnetization in a superconducting coil. Magnetic micropatterns are subsequently produced using Thermo-Magnetic Patterning (TMP) and observed by magnetooptical imaging.

In the second part, microfluidic systems are designed and developed. Simple channels are designed based on standard photolithography and soft lithography. Micromagnets developed in the first part are also integrated into microfluidic systems.

The third part consists of using the developed micromagnets for model applications of real biological applications. Magnetic micro and nanoparticles are used as a model for biological elements. At first, particles are simply captured by the micromagnets and their distribution is observed in bright field microscopy. A few characteristics of microfluidics, namely the laminar flow regime and the parabolic flow profile, are introduced experimentally with standard microfluidic channels. Micromagnetic films integrated into microfluidic channels are used to capture and sort magnetic and non-magnetic particles in liquid solution.

This lab practical aims to introduce the students to basic concepts in different fields:

- Synthesis of magnetic materials - Physical Vapor Deposition technique;
- Synthesis of polymeric microfluidic channels (photolithography, soft lithography, polydimethylsiloxane $\{$ PDMS $\}$, plasma bonding);

- Magnetism at low scales (micro-particles, micromagnets, superparamagnetism);

- Microfluidics (flow regimes, flow control);

- Dynamic particle-fluid-magnet interactions.

The students should have a prior knowledge of materials, clean room fabrication and manipulation. Basic magnetism, fluidic and lithography knowledge is also appreciated.

In the case were biological preparation facilities are available, a fourth part can be added in which the students perform functionalization of biological elements with magnetic particles and manipulate them using micromagnets with and without using microfluidics.

The evaluation is preferentially performed in the form a scientific article that will be written by groups of three or four students.

\section{PART 1}

\section{1-1 Deposition of thick hard magnetic films}

\section{Physical Vapor Deposition (PVD) by triode} sputtering system: the principle

In a High Vacuum (HV) chamber, an association of pumps maintains a certain pressure (down to $5.10^{-8}$ mbar). A continuous flux of gas (argon, Ar) is then injected into the chamber to reach a pressure of around $10^{-3} \mathrm{mbar}$. A filament, sufficiently heated by the Joule effect, constitutes a source of electrons $\left(e^{-}\right)$. The emitted electrons 
are strongly attracted by an anode and their trajectory is focused by a magnetic field. During transfer, residual $\mathrm{Ar}$ atoms from the ambient gas collide with electrons. A plasma with ionized species is formed (Argon ions $\left(\mathrm{Ar}^{+}, \mathrm{Ar}^{2+} \ldots\right.$ ) and $\mathrm{e}^{-}$ ). A negatively polarized target attracts the $\mathrm{Ar}^{+}$ ions. Ions, electrons and atoms from the target are then sputtered in all directions, part of them being deposited on a substrate facing the target. Growth of a layer occurs on the substrate. ${ }^{[1-4]}$

As in most PVD techniques, many parameters are relevant and can be adjusted:

- Base pressure: $10^{-6} \mathrm{mbar}$

- Sputtering pressure: $10^{-3} \mathrm{mbar}$

- Target rotation during deposition

- Deposition rate: up to $20 \mu \mathrm{m} / \mathrm{h}$

- Target surface: up to $10 \times 10 \mathrm{~cm}^{2}$

- Substrate temperature: up to $800^{\circ} \mathrm{C}$

\section{Experimental work:}

- Identify the different elements of the triode sputtering system.

- Fix a 4-inch silicon wafer on the substrate holder.

- Start the pump and wait for a sufficiently low pressure to be reached.

- Deposit and anneal a magnetic film: $\{\mathrm{Ta}(100 \mathrm{~nm})$ / NdFeB $(2 \mu \mathrm{m})$ / Ta (100 nm)\}.

- Remove the deposited film from the sputtering machine and magnetize it to saturation.

\section{1-2 Development and observation of magnetic micropatterns}

\section{Magnetic properties}

The remanent magnetization, or remanence, of a material is its magnetization in the absence of an external magnetic field. Remanence is usually noted $M_{R}$.

The coercive field, or coercivity, also noted $\mathrm{H}_{\mathrm{C}}$, of a ferro/ferrimagnetic material corresponds to the magnetic field which needs to be applied to the material in order to reduce its net magnetization to zero. When the coercivity is high, the material is called a "hard magnet". Typical values of the coercivity for hard magnets are about $10^{5} \mathrm{~A} / \mathrm{m}$ to $2.10^{6} \mathrm{~A} / \mathrm{m}$.

The value of the magnetic susceptibility $\left(\chi_{m}\right)$ of a material gives an indication of its response in the presence of an external magnetic field. Materials are classified as paramagnetic, superparamagnetic, diamagnetic or ferromagnetic, according to their susceptibilities.
The magnetization $\mathrm{M}$ of the material in the presence of a field $\mathrm{H}$ is given by

$$
M=\chi H
$$

Diamagnetic materials are the only ones to present a negative susceptibility, meaning that their induced magnetization is anti-parallel to the applied field.

\section{Thermo-Magnetic Patterning (TMP) ${ }^{[5]}$}

The basic idea, here, is to exploit the temperature dependence of coercivity to reorient the magnetization of a material using an external magnetic field. The principle is similar to bit-by-bit writing of magnetic recording media.

During this lab practical a nanosecond pulsed laser will be used to heat the layer. A hard magnetic film is irradiated with a $\mathrm{KrF}(248 \mathrm{~nm})$ pulsed excimer laser during 20 nanoseconds in the presence of an external magnetic field of $\sim 0.4$ $\mathrm{T}$ created by a bulk magnet. The TMP process is schematized in Fig. 1.

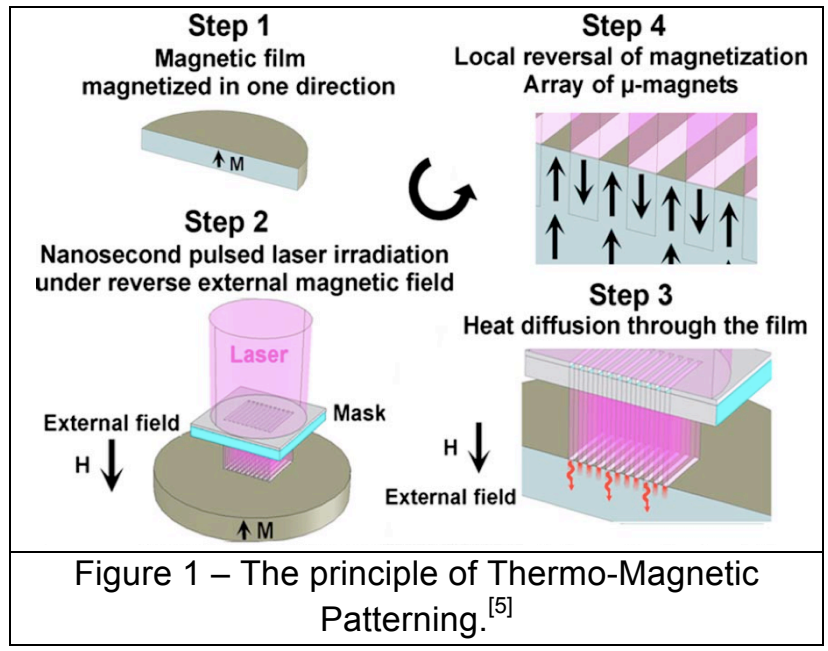

Step 1: The hard magnetic film is initially saturated in one direction.

Step 2: The film is then placed in an external magnetic field and locally heated by irradiation through a mask with characteristic dimensions in the range of 5-100 $\mu \mathrm{m}$. During irradiation, the mask is placed on the film and a magnetic field is applied, opposing the initial magnetization of the film.

Note: The applied field $\mu_{0} H_{\text {app }}$ is such that the total field $\mu_{0} H_{\text {tot }}$ (applied field + demagnetizing field), acting on the magnetic film is less than its 
coercivity at room temperature $\mu_{0} H_{c}(300 \mathrm{~K})$. In this case the magnetization of the film is not reoriented when it is placed in the external field.

Step 3: The temperature at the surface of the film quickly rises and heat diffuses through the film.

Step 4: In the regions heated up to a temperature $T$ such that $\mu_{0} H_{\text {tot }}$ overcomes $\mu_{0} H_{c}(T)$, magnetization reversal occurs. The resulting structure consists of an array of oppositely magnetized micromagnets.

\section{Experimental work:}

- Scribe a guideline on the silicon substrate (not on the magnetic film) using a diamond tip. Choose sample dimensions adapted to the laser spot or to the final system. In this case, the microfluidic system will require samples which are $20 \times 20$ $\mathrm{mm}^{2}$. Cut the film by cleaving it along the guideline.

Note: Silicon wafers have preferential breaking directions that are related to their crystalline structure. These directions are either parallel or perpendicular to the biggest chamfer on the wafer.

- Adjust the laser fluence, not to damage the film (Fluence $=$ Energy $/$ Surface $\left[\mathrm{J} / \mathrm{cm}^{2}\right]$ ).

- Produce magnetic micropatterns using different masks (chessboards and lines). Be careful with the relative direction of magnetization of the bulk magnet and the magnetic film.

\section{1-3 Characterization of the magnetic patterns}

\section{Magneto-Optical characterization: The principle}

A MOIF (Magneto-Optical Indicator Film) is a soft magnetic film used to reveal local variations in the direction of magnetization of a magnetic field. The MOIF used in the practical consists of a garnet layer with a thin, reflective aluminum layer on one side.

The MOIF is magnetically polarized by the micromagnets that are located directly below it. Polarized light irradiates the MOIF, passing through the garnet layer and is reflected by the aluminum layer. The light passes through the film a second time. Upon passing through the film, the polarization of the light rotates due to the Faraday effect, the angle of rotation depending on the magnetic "active" layer of the $\mathrm{MOIF}^{[6]}$. The principle is schematically depicted in Fig. 2 .

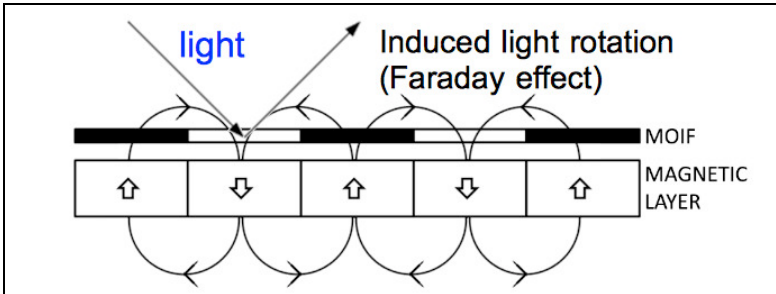

Figure 2 - Principle of a Magneto-Optical Indicator Film (MOIF)

The light is then collected by the ocular of the optical microscope. A magnetic contrast appears, revealing a direct image of the stray field created by the micromagnets on the MOIF.

\section{Experimental work:}

- Using an optical microscope with polarized light, have a direct look at the surface of the sample. Do you see any changes? (If the fluence of the laser is correctly adjusted, the surface of the film should not present any difference from the non-patterned state).

- Position a MOIF directly on the sample surface, with the reflective layer touching the magnetic film. What do you see? (An image of the stray field is visualized at this state). How can we know that it is magnetic contrast?

\section{PART 2}

\section{2-1 Design of microfluidic systems}

In the design of a micro-channel the students have to consider how many inlets and outlets will be needed and what is the volume of interest. In the present case they will work with:

(a) a solution which will be flown individually through the channel $\rightarrow$ one inlet and one outlet;

(b) two solutions which will be flown simultaneously through the channel $\rightarrow$ two inlets and two outlets.

Given that in the first case the goal is to trap magnetic objects over a large surface on the bottom of the channel, the channel should be wide and not very high. The length will be adapted in order to give the largest surface possible. Examples of channels are shown in Figure 3. 


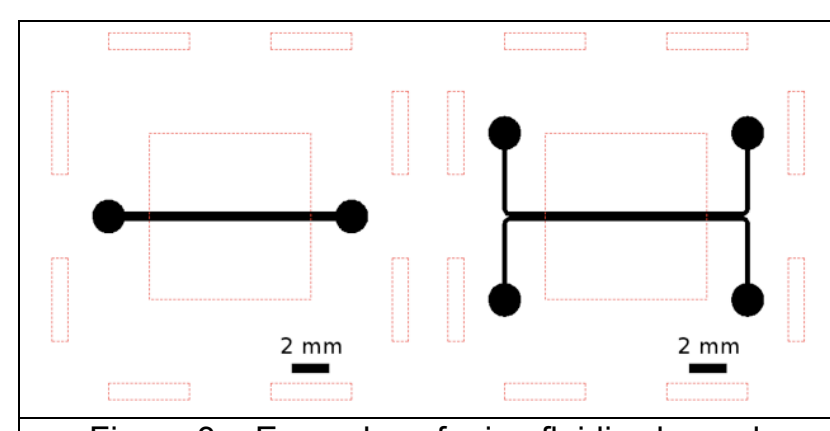

Figure 3 - Examples of microfluidic channel designs. In black, the channels. The rectangles indicate the edges of the microsystem, while the square indicates where the magnetic patterns will be.

Once the dimensions are defined, the channels are drawn with the aid of appropriate software and the resulting file printed on a plastic sheet, preferentially using a high-resolution printer. This sheet will be the "mask" used for photolithography.

Note: A few commonly used softwares are Cadence (shareware), CleWin (shareware), AutoCAD (shareware) or LibreCAD (freeware).

\section{2-2 Development of molds for microchannels}

A dry film photoresist will be used to create the molds for the micro-channels. Liquid photoresists may be used, but processing is longer and more complicated. Dry film photoresist are films with two adhesive surfaces, which are protected by transparent plastic films. The film will be applied on a glass plate, locally exposed to UV light and developed, resulting in a mold for the microchannels.

Note: A positive or negative photoresist can be used, according to the mask. Inversely, the mask design can be adapted according to the available photoresist.

\section{Experimental work:}

- Take a standard glass slide and clean it with acetone, then with isopropanol. Use a compressed air pistol to dry the isopropanol on the slide, trying not to leave any stains.

- Remove the plastic cover from one side of the dry film photoresist and apply it on the glass plate, sticking it to the edge of the plate and gently sliding a finger on it to stick the rest. Avoid creating air bubbles between the film and the glass slide. DO NOT REMOVE THE SECOND PLASTIC COVER YET.

- Position the mask corresponding to the microfluidic channel on top of the photoresist and expose it to UV light. The time and exposing dose are defined according to the photoresist used.

- Remove the second plastic cover.

- Develop the photoresist with the appropriate developer (often for dry film photoresists it is a solution of water with $1 \%$ wt. $\mathrm{Na}_{2} \mathrm{CO}_{3}$ ) and wash it thoroughly with flowing water. Dry it using an air pistol.

\section{2-3 Development of microchannels and integration of micromagnets}

Liquid PDMS (from the Sylgard 184 Elastomer Kit) is poured onto the molds. In order to eventually obtain leak-proof microchannels by plasma bonding, a PDMS layer is spin-coated on the magnetic films. The thickness of this layer is adjusted according to the rotation speed and duration of spin-coating. Note that this layer does not have to be applied on all the films, since the capture of particles will also be performed without microfluidics.

The channels are sealed by exposing both the PDMS part containing the microchannel and the magnetic film with a PDMS layer to oxygen plasma. This process creates a strong chemical bond between the polymeric chains on the two parts, which resists to strong pressure applied to the microchannel.

The system used to observe the laminar flow regime and the parabolic flow profile do not necessarily have to be bonded to the magnetic films. These can be bonded to a glass slide, also using an oxygen plasma.

\section{Experimental work:}

- Put the necessary amount of the non-crosslinked PDMS in a beaker (around $10 \mathrm{ml}$ for one microchannel) and add one tenth of this amount of crosslinking agent.

- Mix both liquids vigorously using a glass rod.

- Degas the solution in a vacuum chamber.

- Spin-coat a layer of PDMS onto the magnetic films.

- Put the molds in a container, ideally a flexible plastic box and pour PDMS on them. 
- Place both the films and the molds in a furnace at $50^{\circ} \mathrm{C}$.

Note: The crosslinking reaction in PDMS can be accelerated by heating. It takes approximately 20 minutes for PDMS to harden at $100{ }^{\circ} \mathrm{C}, 2$ hours at $50{ }^{\circ} \mathrm{C}$ and two to three days at room temperature. The use of excessive temperature can demagnetize the magnets. Heating the magnets to $50{ }^{\circ} \mathrm{C}$ does not have a significant impact on their magnetization.

- Once the PDMS is hardened, remove it from the molds and cut it to approximately the same size as the substrate film.

- Drill holes in the PDMS, on the inlet and outlet locations in order to access the micro-channel. Remove any debris remaining on the pieces.

- Place both the PDMS part containing the microchannel (the microchannel facing up) and a glass plate or the magnetic film in an oxygen plasma chamber. Exposure to plasma at $50 \mathrm{~W}$ for $20 \mathrm{~s}$ at a pressure of around $10^{-3} \mathrm{mbar}$. If it does not, try to adjust the parameters in order to obtain a strong and homogeneous bond.

\section{PART 3}

\section{3-1 Capturing and positioning magnetic particles}

\section{Magnetic forces}

A magnetic field gradient is required in order to exert a translational force on a magnetic object. A uniform field induces a torque, but no translational force $^{[7]}$.

The force acting on a point-like magnetic object is given by

$$
F_{m}=\mu_{0} V_{n p} M \nabla H
$$

where $\mu_{0}$ is the vacuum permeability, $V_{n p}$ is the volume of the object, $M$ its magnetization and $\nabla \mathrm{H}$ the gradient of magnetic field ${ }^{[8]}$. In the case of superparamagnetic particles, the magnetization is given by

$$
M=M_{0} L(x)=M_{0}[\operatorname{coth}(x)-1 / x]
$$

with

$$
x=\frac{\mu_{0} V_{n p} M_{0} H}{k_{B} T}
$$

where $M_{0}$ is its saturation magnetization, $k_{B}$ is Boltzmann's constant and $T$ is the temperature.

As it can be deduced from the equation, the force increases in the zones of highest magnetic field gradient. Thus, particles are expected to be attracted to the most inhomogeneous zones, as regards the magnetic field, i.e., the zones close to the interfaces between oppositely oriented magnets. ${ }^{[9]}$

In this part the students will observe how magnetic particles are distributed after being put on a patterned magnet in liquid solution. No microfluidic channels are needed. A droplet of the solution containing magnetic particles can be put on a magnetic film, or the film can be submerged in a container with the solution.

Biological studies can be performed in this part, as reported in [10].

\section{Experimental work:}

- Mix magnetic micro and nanoparticles with water in order to obtain a diluted solution. Commercial magnetic particles are usually supplied in a very concentrated solution, which is not adapted for manipulation.

- With a pipette, put droplets of the solution on the patterned magnetic film. How do the particles behave in the presence of magnetic micropatterns? Why? Is it in agreement with the theory?

\section{3-2 Microfluidics: the basics}

\section{Flow regimes and the Reynolds number}

A laminar flow (or a flow in the laminar regime) is the type of flow in which the layers of fluid are submitted to a minimum agitation. Fluid streams tend to flow parallel, without mixing (no swirls or eddies). In this regime the viscosity of the fluid is much more important than its kinetic energy. The fluid flows with maximum velocity in the center of the channel, while the velocity is considered to be zero at the edges of the pipe/channel.

A turbulent flow is considered to be the opposite case: the flow regime is chaotic, containing vortexes and eddies, which makes the flow unpredictable.

A transitional flow is characterized by a mixture of both laminar and turbulent flows: the fluid flows in 
a laminar regime near the edges of the channel, while a turbulent regime is present at the center. The dimensionless Reynolds number $(\mathrm{Re})$ is often used to identify flow regimes. It presents the relative significance of viscous effects as compared to inertial effects. Its calculation is based on channel dimensions, fluid properties and system conditions. The different flow regimes, and their corresponding range of Reynolds numbers is shown below:

\begin{tabular}{l|l} 
Reynolds number & Flow regime \\
\hline $\operatorname{Re}<2300$ & Laminar \\
$2300<\operatorname{Re}<4000$ & Transitional \\
$\operatorname{Re}>4000$ & Turbulent
\end{tabular}

\section{Microfluidic Flow Control System}

A Microfluidic Flow Control System (MFCS) is a device that allows the user to precisely control either the pressure or the flow rate on specific points of a microfluidic system. The working principle of an MFCS is depicted in Fig. 4. A graphic user interface (GUI) allows the user to control the pressure channels of the equipment. The outlet of each channel is connected to the container in which the sample solution is stored. Once the pressure is increased in the tube, the solution is pushed out through the outlet of the container. These outlets can be connected to a flowmeter (which will measure the flow rate, usually in $\mu \mathrm{l} / \mathrm{min}$ ) and then to the inlets and outlets of the microfluidic channel.

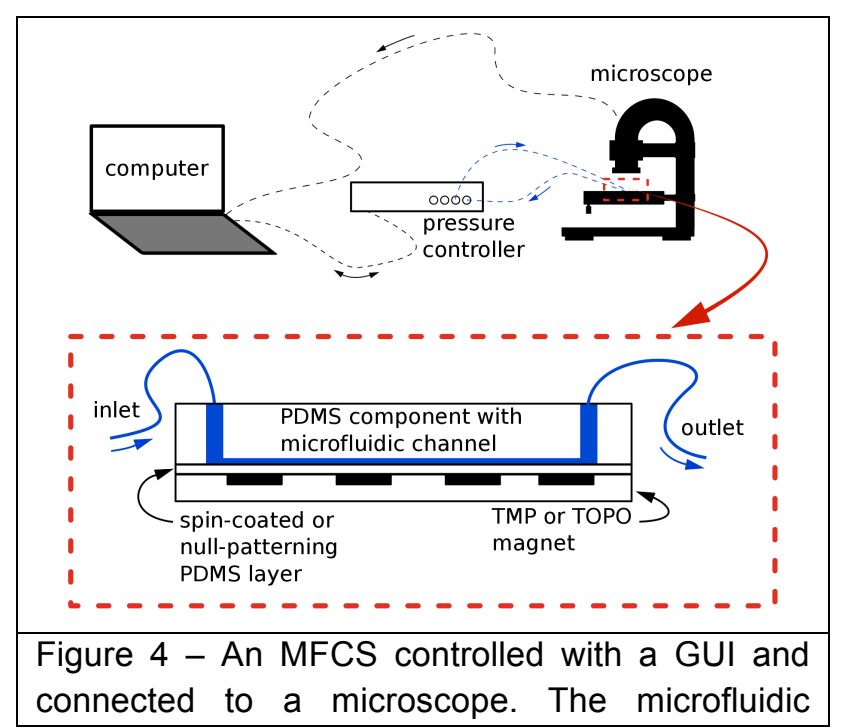

system connected to the different channels can be observed in the zoom.

Syringe pumps can also be used to control the flow in microfluidics. In a syringe pump, the piston of a syringe is pushed or pulled at a known rate. Knowing the dimensions of the syringe allows the user to calculate the corresponding flow rate. Syringe pumps are much simpler and cheaper than pressure controllers, however their response time and precision is significantly lower and can be limiting for many applications.

In this part the students connect up the microfluidic system to a pressure controller or syringe pump and observe liquids flowing in simple channels using a microscope. The parabolic flow profile is observed by dispersing non-magnetic particles in a liquid and measuring their velocity in the channel. A demonstration of the laminar flow regime is performed using liquids of two different colors flown at the same time in the channel without mixing.

\section{Experimental work:}

- Connect the inlets and outlets of the system to the according channel of a pressure controller or a syringe pump.

- Place a mixture of water and non-magnetic particles in a container.

- Pump the mixture through the channel and observe how the liquid flows.

- Make a video in which the particles can be seen flowing both in the center and along the edges of the channel.

- Use the video to calculate an approximate flow velocity for particles at different points along the channel. Is there a difference in velocity between the points? What are the limitations of this technique to follow profile observations?

Note: If a confocal microscope is used, a good approximation of flow velocity can be obtained. In standard microscopy, however, particles flowing at different heights in the channel are seen, thus giving different flow velocities for the same streamline.

- Connect the channel with two inlets and two outlets to the flow controller.

- Place two mixtures of different colors (dilute a food dye in water, for example) in different containers. 
- Pump both liquids through the channel at the same time. How do the liquids flow? Is there a difference between the region close to the inlets and the one close to the outlets?

- Modify, independently of each other, the applied pressures or flow rates on each inlet. What is the effect on the flowing liquids?

\section{3-3 Magnetic sorting using microfluidics}

\section{Magnetic sorting}

Magnetic sorting exploits the difference in the balance of forces acting, on the one hand, on nonmagnetic objects submitted to gravity (including buoyancy) and viscous forces (neglecting Brownian motion) and, on the other hand, on magnetic objects (e.g. magnetic particles), additionally submitted to a magnetic force. Gravity forces are expressed as

$$
F_{g}=\frac{4}{3} \pi r_{\text {part }}^{3}\left(\rho_{\text {part }}-\rho_{\text {medium }}\right) g
$$

where $\rho_{\text {part }}$ is the particle mass density, $\rho_{\text {medium }}$ is the medium mass density and $g$ is the gravitational acceleration. For a particle of radius $r_{\text {part }}$ flowing with velocity $v_{\text {part }}$ in a medium of viscosity $\eta$ and velocity $v_{\text {fluid, }}$, the drag force is given by

$$
F_{d}=6 \pi \eta r_{\text {part }}\left(v_{\text {medium }}-v_{\text {part }}\right) .
$$

For the cases considered here, the inertia of the particles can be neglected due to the time scale of inertial events compared to other movements of the particles. Thus, the particles can be considered to move at their limiting velocity given by

$$
\begin{aligned}
& v_{x}=\frac{F_{m x}}{6 \pi \eta r_{\text {part }}} \\
& v_{y}=v_{\text {fluid }}+\frac{F_{m y}}{6 \pi \eta r_{\text {part }}} \\
& v_{z}=\frac{F_{g}+F_{m z}}{6 \pi \eta r_{\text {part }}}
\end{aligned}
$$

where $F_{m x}, F_{m y}$ and $F_{m z}$ are the components of the magnetic force along the $\mathrm{x}, \mathrm{y}$ and $\mathrm{z}$-axis, respectively. The displacement of the particles is thus derived using $d x / d t=u_{x}(t), d y / d t=u_{y}(t)$ and $d z / d t=u_{z}(t)^{[10]}$.

The students perform magnetic sorting in microfluidics in this part of the practical work. At first a channel with one inlet and one outlet, with integrated chessboard-like magnetic patterns, is used to capture magnetic particles, while nonmagnetic particles flow through the channel.

Note: magnetic and non-magnetic particles should be distinguishable in the channel, either by different colors of fluorescence of using colored particles.

Then a channel with two inlets and two outlets is used to perform continuous flow magnetic sorting. A solution of magnetic and non-magnetic particles is pumped through one inlet of the channel, while a buffer liquid is pumped through the other. Magnetic rails disposed in the channel with a certain angle related to the fluid flow guide the magnetic particles to the other side of the channel, while the non-magnetic particles remain in the initial streamline (see Fig. 5).

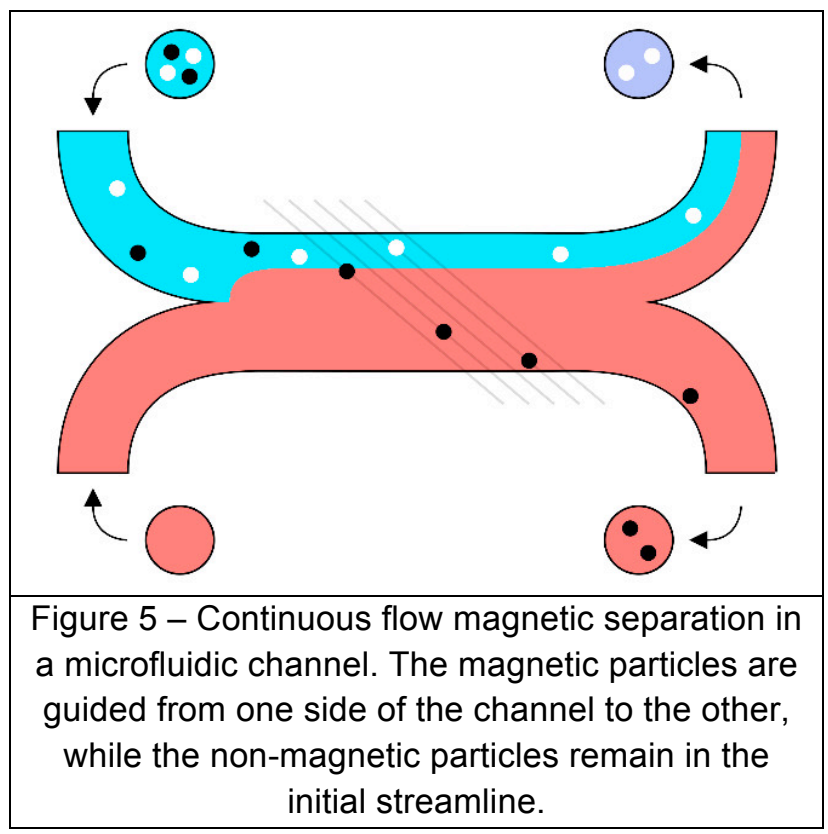

\section{Experimental work:}

- Connect the inlet and outlet of the system to the according channels of a pressure controller or a syringe pump.

- Place a mixture of non-magnetic and magnetic particles diluted in water in a container.

- Pump the mixture through the channel and observe how the particles flow.

- Adjust the pressure/flow rate so that magnetic 
sorting can be performed. What is the expected behavior of each type of particle in the channel? Is this observed experimentally? What are the limitations of this type of magnetic sorting?

- Make a video in which magnetic sorting can be observed.

Note: The efficiency of sorting can be calculated using a technique of particle counting such as flow cytometry.

- Connect the channel with two inlets and two outlets to the flow controller.

- Place a mixture of magnetic and non-magnetic particles in a container and water in another one. The solutions can be dyed for easier visualization of flows.

- Pump both liquids through the channel at the same time, trying to focus the solution with the particles on a very small part of the channel.

- Adjust the flow rates so that guiding of magnetic particles can be performed. How do the particles flow? How can this technique be useful, as compared to magnetic sorting as performed in the previous experiment?

- Make a video in which particle guiding can be observed.

\section{Discussion and conclusions}

This lab practical should allow students to have first contact with basic and advanced techniques of microfabrication and manipulation.

The first part concerning the deposition by PVD of thick magnetic films and the laser-based technique for the development of micromagnets are both cutting-edge technologies.

The second part is based on standard techniques and materials, developed in a cleanroom environment. The technical knowledge brought to the students by this part is commonplace, yet a requirement for any person working in the field, since it is part of the basis for the fabrication of the more sophisticated devices.

Students should be able to carry out this lab practical with minimum guidance and help, so that they can be fully aware of all the constraints in the design and used of the microsystem.

As an ensemble, this lab practical has a rich range of domains in which the students are guided to think for themselves, question their decisions and promptly act to correct eventual mistakes and misconceptions.

The suggested evaluation format, an article-like report, is a good stimulus for a previous planning of the experimental work and a strong exercise in synthesis of data and organization. Autonomy and ability to produce and analyze results are strong points of this lab practical.

The applications can be expanded based on [11].

\section{References}

1 - R. P. Allen, S. D. Dahlgren, H. W. Arrowsmith, J. P. Heinrich, Technical report AFML-TR-74-87 (1974)

2 - B. A. Kapitanov, N. V. Kornilov, Ya. L. Linetsky and V. Yu. Tsvetkov, J. Magn. Magn. Mater. 127, 289 (1993)

3 - N.M. Dempsey, A. Walther, F. May, D. Givord, K. Khlopkov and O. Gutfleisch, Appl. Phy. Lett. 96, 092509 (2007)

4 - A. Walther, K. Khlopkov, O. Gutfleisch, D. Givord and N. M. Dempsey, J. Magn. Magn. Mater. 316, 174 (1993)

5 - F. Dumas-Bouchiat, L. F. Zanini, M. Kustov, N. M. Dempsey, R. Grechishkin, K. Hasselbach, J. C. Orlianges, C. Champeaux, A. Catherinot and D. Givord, Appl. Phys. Lett. 96,102511 (2010) 6 - R. Grechishkin, S. Chigirinsky, M. Gusev, O. Cugat and N. M. Dempsey, In: Asti, Pareti and Ghidini (eds) Mag. Nanostruc. in Mod. Tech., pp. 195-224. Springer, Netherlands (2007)

7 - M. A. M. Gijs, F. Lacharme and U. Lehmann, Chem. Rev. 110, 1518 (2010)

8 - M. Kustov, P. Laczkowski, D. Hykel, K. Hasselbach, F. Dumas-Bouchiat, D. O'Brien, P. Kauffmann, R. Grechishkin, D. Givord, G. Reyne, O. Cugat and N. M. Dempsey, J. Appl. Phys. 108, 1 (2010)

9 - L. F. Zanini, O. Osman, M. Frenea-Robin, N. Haddour, N. M. Dempsey, G. Reyne and F. Dumas-Bouchiat, J. App. Phys, 111, 07 B312 (2012)

10 - O. Osman, L. F. Zanini, M. Frénéa-Robin, F. Dumas-Bouchiat, N. M. Dempsey, G. Reyne, F. Buret and N. Haddour, Biomed. Microdev., 14, 947 (2012)

11 - L.F. Zanini, N. M. Dempsey, D. Givord, G. Reyne and F. Dumas-Bouchiat, Appl. Phys. Lett. 99, 232504 (2011) 